\title{
Attacking Reduced-Round Versions of the SMS4 Block Cipher in the Chinese WAPI Standard*
}

\author{
Jiqiang Lu \\ Information Security Group, Royal Holloway, University of London \\ Egham, Surrey TW20 0EX, UK \\ lvjiqiang@hotmail.com
}

\begin{abstract}
SMS4 is a 32-round block cipher with a 128-bit block size and a 128-bit user key. It is used in WAPI, the Chinese WLAN national standard. In this paper, we present a rectangle attack on 14-round SMS4, and an impossible differential attack on 16-round SMS4. These are better than any previously known cryptanalytic results on SMS4 in terms of the numbers of attacked rounds.
\end{abstract}

Keywords: Block cipher, SMS4, Impossible differential cryptanalysis, Rectangle attack.

\section{Introduction}

The Chinese national standard for Wireless Local Area Networks (WLANs), WLAN Authentication and Privacy Infrastructure (WAPI), has been the subject of extensive international debate, especially between China and USA, since over the last four years it has been a rival for IEEE 802.11i [6] for adoption as an ISO (International Organization for Standardization) international standard. WAPI and IEEE 802.11i have both been proposed as security amendments to the ISO/IEC 8802-11 WLAN standard [7. The two schemes use two different block ciphers for encryption of data: IEEE 802.11i uses the AES 14 cipher, while WAPI uses the SMS4 1] cipher. In March 2006, IEEE 802.11i was approved as the standard, and WAPI was rejected, partially because of uncertainties regarding the security of the undisclosed SMS4 cipher. However, because it is a Chinese national standard, WAPI continues to be used in the Chinese WLAN industry, and many international corporations, such as SONY, support WAPI in relevant products.

The SMS4 cipher was released in a Chinese version only, in January 2006 [1; it has a 128-bit block size, a 128-bit user key, and a total of 32 rounds. To the best of our knowledge, the only previously published cryptanalytic result on the SMS4 algorithm is an integral attack 9] on 13-round SMS4, presented recently in [10]; moreover, a differential fault analysis on the SMS4 implementation was presented in [16].

In this paper, we exploit certain 12-round rectangle distinguishers with probability $2^{-237.64}$, which can be used to mount a rectangle attack on SMS4 reduced

* This work as well as the author was supported by a British Chevening / Royal Holloway Scholarship and the European Commission under contract IST-2002-507932 (ECRYPT).

S. Qing, H. Imai, and G. Wang (Eds.): ICICS 2007, LNCS 4861, pp. 306-318, 2007.

(C) Springer-Verlag Berlin Heidelberg 2007 
to 14 rounds. We also exploit certain 12-round impossible differentials, which enables us to mount an impossible differential attack on SMS4 reduced to 16 rounds. The attacks use the early abort technique described in 11 12 13.

The rest of this paper is organised as follows. In the next section, we describe the notation used throughout this paper and the SMS4 cipher. In Section 3, we introduce a number of properties of SMS4 and give some necessary definitions. In Sections 4 and 5, we present our cryptanalytic results. Section 6 concludes this paper.

\section{Preliminaries}

\subsection{Notation}

We use the following notation throughout this paper.

$-\oplus$ : bitwise logical exclusive OR (XOR)

- $i$ : left rotation by $i$ bits

$-e_{j}$ : a 32-bit word with zeros in all positions but bit $j,(0 \leq j \leq 31)$

$-e_{i_{1}, \cdots, i_{j}}: e_{i_{1}} \oplus \cdots \oplus e_{i_{j}},\left(0 \leq i_{1}, \cdots, i_{j} \leq 31\right)$

- ? : an arbitrary 32-bit word, where two words represented by the ? symbol may be different

The notion of difference used throughout this paper is with respect to the $\oplus$ operation. It is assumed that the least significant bit of a 32-bit word is referred as the 0 -th bit and the most significant bit is referred as the 31 st bit.

\subsection{The SMS4 Cipher}

The SMS4 [1] block cipher takes as an input a 128-bit plaintext $P$, represented as four 32 -bit words $P=\left(P_{0}, P_{1}, P_{2}, P_{3}\right)$, and has a total of 32 rounds. Let $X^{i+1}=\left(X_{i+1,0}, X_{i+1,1}, X_{i+1,2}, X_{i+1,3}\right)$ denote the four-word output of the $i$-th round, $(0 \leq i \leq 31) \sqrt{1}$. Then, the encryption procedure of SMS4 is as follows:

1. Set $X^{0}=\left(X_{0,0}, X_{0,1}, X_{0,2}, X_{0,3}\right)=\left(P_{0}, P_{1}, P_{2}, P_{3}\right)$.

2. For $i=0$ to 31 :

$$
\begin{aligned}
& -X_{i+1,0}=X_{i, 1}, \\
& -X_{i+1,1}=X_{i, 2}, \\
& -X_{i+1,2}=X_{i, 3}, \\
& -X_{i+1,3}=X_{i, 0} \oplus \mathrm{L}\left(\mathrm{S}\left(X_{i, 1} \oplus X_{i, 2} \oplus X_{i, 3} \oplus R K_{i}\right)\right),
\end{aligned}
$$

3. The ciphertext is $X^{32}=\left(X_{32,0}, X_{32,1}, X_{32,2}, X_{32,3}\right)$,

where $R K_{i}$ is the 32 -bit round subkey for the $i$-th round, the transformation $\mathrm{L}$ is defined as $\mathrm{L}(x)=x \oplus(x \lll 2) \oplus(x \lll 10) \oplus(x \lll 18) \oplus(x \lll 24)$, for $x \in Z_{2}^{32}$, and the transformation $\mathrm{S}$ applies the same $8 \times 8$ bijective S-Box (see Table 1) four times in parallel to an input, and it is defined as follows.

$$
\begin{aligned}
& \text { input: } A=\left(a_{0}, a_{1}, a_{2}, a_{3}\right) \in\left(Z_{2}^{8}\right)^{4} \text {, output: } B=\left(b_{0}, b_{1}, b_{2}, b_{3}\right) \in\left(Z_{2}^{8}\right)^{4} \\
& \text { substitution : } B=\mathrm{S}(A) \Leftrightarrow b_{j}=\mathrm{S}-\operatorname{Box}\left(a_{j}\right) \text {, for } j=0,1,2,3 .
\end{aligned}
$$

\footnotetext{
${ }^{1}$ Note that the first round is referred as Round 0.
} 
Table 1. The S-Box table of SMS4

\begin{tabular}{|l||cccccccccccccccccc|c|}
\hline & $0 x 0$ & $0 x 1$ & $0 x 2$ & $0 x 3$ & $0 x 4$ & $0 x 5$ & $0 x 6$ & $0 x 7$ & $0 x 8$ & $0 x 9$ & $0 x a$ & $0 x b$ & $0 x c$ & $0 x d$ & $0 x e$ & $0 x f$ \\
\hline \hline $0 x 0$ & $d 6$ & 90 & $e 9$ & $f e$ & $c c$ & $e 1$ & $3 d$ & $b 7$ & 16 & $b 6$ & 14 & $c 2$ & 28 & $f b$ & $2 c$ & 05 \\
\hline $0 x 1$ & $2 b$ & 67 & $9 a$ & 76 & $2 a$ & $b e$ & 04 & $c 3$ & $a a$ & 44 & 13 & 26 & 49 & 86 & 06 & 99 \\
\hline $0 x 2$ & $9 c$ & 42 & 50 & $f 4$ & 91 & $e f$ & 98 & $7 a$ & 33 & 54 & $0 b$ & 43 & $e d$ & $c f$ & $a c$ & 62 \\
\hline $0 x 3$ & $e 4$ & $b 3$ & $1 c$ & $a 9$ & $c 9$ & 08 & $e 8$ & 95 & 80 & $d f$ & 94 & $f a$ & 75 & $8 f$ & $3 f$ & $a 6$ \\
\hline $0 x 4$ & 47 & 07 & $a 7$ & $f c$ & $f 3$ & 73 & 17 & $b a$ & 83 & 59 & $3 c$ & 19 & $e 6$ & 85 & $4 f$ & $a 8$ \\
\hline $0 x 5$ & 68 & $6 b$ & 81 & $b 2$ & 71 & 64 & $d a$ & $8 b$ & $f 8$ & $e b$ & $0 f$ & $4 b$ & 70 & 56 & $9 d$ & 35 \\
\hline $0 x 6$ & $1 e$ & 24 & $0 e$ & $5 e$ & 63 & 58 & $d 1$ & $a 2$ & 25 & 22 & $7 c$ & $3 b$ & 01 & 21 & 78 & 87 \\
\hline $0 x 7$ & $d 4$ & 00 & 46 & 57 & $9 f$ & $d 3$ & 27 & 52 & $4 c$ & 36 & 02 & $e 7$ & $a 0$ & $c 4$ & $c 8$ & $9 e$ \\
\hline $0 x 8$ & $e a$ & $b f$ & $8 a$ & $d 2$ & 40 & $c 7$ & 38 & $b 5$ & $a 3$ & $f 7$ & $f 2$ & $c e$ & $f 9$ & 61 & 15 & $a 1$ \\
\hline $0 x 9$ & $e 0$ & $a e$ & $5 d$ & $a 4$ & $9 b$ & 34 & $1 a$ & 55 & $a d$ & 93 & 32 & 30 & $f 5$ & $8 c$ & $b 1$ & $e 3$ \\
\hline $0 x a$ & $1 d$ & $f 6$ & $e 2$ & $2 e$ & 82 & 66 & $c a$ & 60 & $c 0$ & 29 & 23 & $a b$ & $0 d$ & 53 & $4 e$ & $6 f$ \\
\hline $0 x b$ & $d 5$ & $d b$ & 37 & 45 & $d e$ & $f d$ & $8 e$ & $2 f$ & 03 & $f f$ & $6 a$ & 72 & $6 d$ & $6 c$ & $5 b$ & 51 \\
\hline $0 x c$ & $8 d$ & $1 b$ & $a f$ & 92 & $b b$ & $d d$ & $b c$ & $7 f$ & 11 & $d 9$ & $5 c$ & 41 & $1 f$ & 10 & $5 a$ & $d 8$ \\
\hline $0 x d$ & $0 a$ & $c 1$ & 31 & 88 & $a 5$ & $c d$ & $7 b$ & $b d$ & $2 d$ & 74 & $d 0$ & 12 & $b 8$ & $e 5$ & $b 4$ & $b 0$ \\
\hline $0 x e$ & 89 & 69 & 97 & $4 a$ & $0 c$ & 96 & 77 & $7 e$ & 65 & $b 9$ & $f 1$ & 09 & $c 5$ & $6 e$ & $c 6$ & 84 \\
\hline $0 x f$ & 18 & $f 0$ & $7 d$ & $e c$ & $3 a$ & $d c$ & $4 d$ & 20 & 79 & $e e$ & $5 f$ & $3 e$ & $d 7$ & $c b$ & 39 & 48 \\
\hline
\end{tabular}

The composed transformation $\mathrm{L} \circ \mathrm{S}$ is called $\mathrm{T}$ in the specification document 1]. Fig. 1 depicts one encryption round of SMS4. Decryption is identical to encryption, except that the round keys are used in the reverse order.

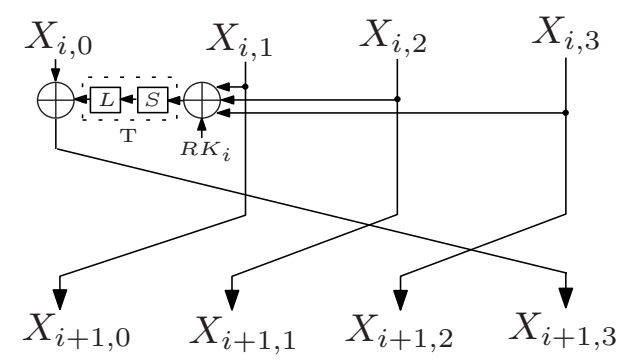

Fig. 1. The $i$-th encryption round of SMS4

The key schedule of SMS4 accepts a 128-bit user key $M K$, represented as four 32-bit words $\left(M K_{0}, M K_{1}, M K_{2}, M K_{3}\right)$. The $j$-th round subkey $R K_{j}(0 \leq j \leq$ $31)$ is generated as follows.

- Compute $\left(K_{0}, K_{1}, K_{2}, K_{3}\right)=\left(M K_{0} \oplus F K_{0}, M K_{1} \oplus F K_{1}, M K_{2} \oplus F K_{2}, M K_{3} \oplus\right.$ $F K_{3}$ ), where $F K_{0}=0 x a 3 b 1 b a c 6, F K_{1}=0 x 56 a a 3350, F K_{2}=0 x 677 d 9197$, and $F K_{3}=0 x b 27022 d c$.

- Compute $R K_{j}=K_{j+4}=K_{j} \oplus \mathrm{L}^{\prime}\left(\mathrm{S}\left(K_{j+1} \oplus K_{j+2} \oplus K_{j+3} \oplus C K_{j}\right)\right)$, where the transformation $\mathrm{L}^{\prime}$ is defined as $\mathrm{L}^{\prime}(x)=x \oplus(x \lll 13) \oplus(x \lll 23)$, for $x \in Z_{2}^{32}$, and the constant $C K_{j}=\left(c k_{j, 0}, c k_{j, 1}, c k_{j, 2}, c k_{j, 3}\right) \in\left(Z_{2}^{8}\right)^{4}$, with $c k_{j, k}=28 j+7 k \bmod 256(k=0,1,2,3)$. The composed transformation $\mathrm{L}^{\prime} \circ \mathrm{S}$ is called $\mathrm{T}^{\prime}$ in the specification document. 


\section{Properties of SMS4 and Definitions}

We first introduce three properties of SMS4, which are important to our attacks.

Property 1. For the nonlinear transformation $\mathrm{S}, \mathrm{S}(\Delta x)=0$ if, and only if, $x=0\left(x \in Z_{2}^{32}\right)$.

Property 2. For the linear transformation L, L $(x)=0$ if, and only if, $x=0$ $\left(x \in Z_{2}^{32}\right)$.

Property 3. For the S-Box, there exist 127 possible output differences for any nonzero input difference, of which 1 output difference occurs with probability $2^{-6}$, and each of the other 126 output differences occurs with probability $2^{-7}$.

Property 1 is obvious; Properties 2 and 3 can be verified by two simple computer programs.

We next give two definitions.

Definition 1. Let $\Lambda$ be an arbitrary but nonempty subset of any of the four sets $\{0,1, \cdots, 7\},\{8,9, \cdots, 15\},\{16,17, \cdots, 23\}$ and $\{24,25, \cdots, 31\}$, then we define the set $\Omega\left(e_{\Lambda}\right)$ as follows:

$$
\Omega\left(e_{\Lambda}\right)=\left\{x \mid x=\mathrm{L}(y), \operatorname{Pr}\left(\mathrm{S}\left(\Delta e_{\Lambda}\right) \rightarrow \Delta y\right)=2^{-6}, x, y \in Z_{2}^{32}\right\} .
$$

Note that $\left|\Omega\left(e_{\Lambda}\right)\right|=1$ holds for any nonempty $\Lambda$ by Property 3 .

Definition 2. Let $\Lambda$ be an arbitrary but nonempty subset of the set $\{0,1, \cdots, 31\}$; then we define the three sets $\Theta\left(e_{\Lambda}\right), \Upsilon\left(e_{\Lambda}, m \in \Theta\left(e_{\Lambda}\right)\right)$ and $\Pi\left(e_{\Lambda}, m \in \Theta\left(e_{\Lambda}\right), n \in\right.$ $\left.\Upsilon\left(e_{\Lambda}, m\right)\right)$ as follows:

- $\Theta\left(e_{\Lambda}\right)=\left\{x \mid x=\mathrm{L}(y), \operatorname{Pr}\left(\mathrm{S}\left(\Delta e_{\Lambda}\right) \rightarrow \Delta y\right)>0, x, y \in Z_{2}^{32}\right\}$.

- $\Upsilon\left(e_{\Lambda}, m \in \Theta\left(e_{\Lambda}\right)\right)=\left\{x \mid x=\mathrm{L}(y) \oplus e_{\Lambda}, y \in\{z \mid \operatorname{Pr}(\mathrm{S}(\Delta m) \rightarrow \Delta z)>0, z \in\right.$ $\left.\left.Z_{2}^{32}\right\}, x, y \in Z_{2}^{32}\right\}$.

- $\Pi\left(e_{\Lambda}, m \in \Theta\left(e_{\Lambda}\right), n \in \Upsilon\left(e_{\Lambda}, m\right)\right)=\left\{x \mid x=\mathrm{L}(y) \oplus e_{\Lambda}, y \in\left\{z \mid \operatorname{Pr}\left(\mathrm{S}\left(\Delta\left(e_{\Lambda} \oplus\right.\right.\right.\right.\right.$ $\left.\left.m \oplus n)) \rightarrow \Delta z)>0, z \in Z_{2}^{32}\right\}, x, y \in Z_{2}^{32}\right\}$.

\section{Rectangle Attack on 14-Round SMS4}

Being a variant of the boomerang attack [15] and an improvement of the amplified boomerang attack [8], the rectangle attack [4] shares the same basic idea of using two short differentials with larger probabilities instead of a long differential with a smaller probability. A rectangle attack is based on a rectangle distinguisher, which treats a block cipher $E:\{0,1\}^{n} \times\{0,1\}^{k} \rightarrow\{0,1\}^{n}$ as a cascade of two sub-ciphers $E=E^{1} \circ E^{0}$.

In this section, we exploit certain 12-round rectangle distinguishers with probability $2^{-237.64}$, such that we can conduct a rectangle attack on SMS4 reduced to that operates 14 rounds. 


\subsection{2-Round Rectangle Distinguishers with Probability $2^{-237.64}$}

Let $E^{0}$ denote Rounds 0 to 7 of SMS4, and $E^{1}$ denote Rounds 8 to 11 of SMS4. The differentials for the 12-round distinguishers are as follows.

- The following 8-round differentials $\alpha \rightarrow \beta^{\prime}$ are used for $E^{0}:\left(e_{\Psi_{1}}, e_{\Psi}, e_{\Psi}, e_{\Psi}\right) \rightarrow$ $\left(e_{\Psi_{2}}, e_{\Psi_{3}}, e_{\Psi_{4}}, e_{\Psi_{5}}\right)$, where $\Psi$ is an arbitrary but nonempty subset of any of the four sets $\{0,1, \cdots, 7\},\{8,9, \cdots, 15\},\{16,17, \cdots, 23\}$ and $\{24,25, \cdots, 31\}$, $e_{\Psi_{1}} \in \Omega\left(e_{\Psi}\right), e_{\Psi_{2}} \in \Theta\left(e_{\Psi}\right), e_{\Psi_{3}} \in \Upsilon\left(e_{\Psi}, e_{\Psi_{2}}\right), e_{\Psi_{4}} \in \Pi\left(e_{\Psi}, e_{\Psi_{2}}, e_{\Psi_{3}}\right)$, and $e_{\Psi_{5}} \in\left\{x \mid x=\mathrm{L}(y) \oplus e_{\Lambda}, y \in\left\{z \mid \operatorname{Prob} .\left(\mathrm{S}\left(\Delta\left(e_{\Psi_{2}} \oplus e_{\Psi_{3}} \oplus e_{\Psi_{4}}\right)\right) \rightarrow \Delta z\right)>0, z \in\right.\right.$ $\left.\left.Z_{2}^{32}\right\}, x, y \in Z_{2}^{32}\right\}$.

- The following 4-round differentials $\gamma \rightarrow \delta^{\prime}$ are used for $E^{1}:\left(e_{\Phi}, e_{\Phi}, e_{\Phi}, 0\right) \rightarrow$ $\left(e_{\Phi}, e_{\Phi}, e_{\Phi}, e_{\Phi_{2}}\right)$, where $\Phi$ is an arbitrary but nonempty subset of any of the four sets $\{0,1, \cdots, 7\},\{8,9, \cdots, 15\},\{16,17, \cdots, 23\}$ and $\{24,25, \cdots, 31\}$, $e_{\Phi_{2}} \in \Theta\left(e_{\Phi}\right)$.

See Table 2 for the details of these two groups of differentials, where the difference in a round is the input difference to this round. The same meaning is used with the differentials in the next section. Note that different $\Psi$ and/or $\Phi$ correspond to different rectangle distinguishers. In the following, we assume $\Psi$ and $\Phi$ are fixed.

Table 2. The two groups of differentials in the 12-round rectangle distinguisher, where $\dagger$ means that the probability is addressed later

\begin{tabular}{|c|c|c|c|c|c|c|c|c|c|c|c|}
\hline $\operatorname{Round}(i)$ & $\Delta X_{i, 0}$ & $\Delta X_{i,}$ & $\Delta X_{i}$ & $\Delta X_{i, 3}$ & Prob. & Round $(i$ & $\Delta X_{i,}$ & $\Delta X_{i}$, & $\Delta X_{i}$ & $\Delta X_{i, 3}$ & Prob. \\
\hline 0 & $e_{\Psi_{1}}$ & $e_{\Psi}$ & $e_{\Psi}$ & $e_{\Psi}$ & $2^{-6}$ & 7 & $e_{\Psi}$ & $e_{\Psi_{2}}$ & $e_{\Psi_{3}}$ & $e_{\Psi_{4}}$ & $\dagger$ \\
\hline 1 & $e_{\Psi}$ & $e_{\Psi}$ & $e_{\Psi}$ & 0 & 1 & output & $e_{\Psi_{2}}$ & $e_{\Psi_{3}}$ & $e_{\Psi_{4}}$ & $e_{\Psi_{5}}$ & 1 \\
\hline 2 & $e_{\Psi}$ & $e_{\Psi}$ & 0 & $e_{\Psi}$ & 1 & 8 & $e_{\Phi}$ & $e_{\Phi}$ & $e_{\Phi}$ & 0 & 1 \\
\hline 3 & $e_{\Psi}$ & 0 & $e_{\Psi}$ & $e_{\Psi}$ & 1 & 9 & $e_{\Phi}$ & $e_{\Phi}$ & 0 & $e_{\Phi}$ & 1 \\
\hline 4 & 0 & $e_{\Psi}$ & $e_{\Psi}$ & $e_{\Psi}$ & \multirow{3}{*}{$\dagger$} & 10 & $\overline{e_{\Phi}}$ & 0 & $e_{\Phi}$ & $\overline{e_{\Phi}}$ & 1 \\
\hline 5 & $e_{\Psi}$ & $e_{\Psi}$ & $e_{\Psi}$ & $e_{\Psi_{2}}$ & & 11 & 0 & $e_{\Phi}$ & $e_{\Phi}$ & $e_{\Phi}$ & $\dagger$ \\
\hline 6 & $e_{\Psi}$ & $e_{\Psi}$ & $e_{\Psi_{2}}$ & $e_{\Psi_{3}}$ & & output & $e_{\Phi}$ & $e_{\Phi}$ & $e_{\Phi}$ & $e_{\Phi_{2}}$ & 7 \\
\hline
\end{tabular}

In the following, we need to sum the square of the probabilities of all the possible differentials $\alpha \rightarrow \beta^{\prime}$. As there exist many more differential characteristics than we can count, it is infeasible to compute the exact square sum; however, we can compute a lower bound for it. By the Property 3 in Section 3 , we can learn that for a fixed $\Psi$, there exists one $e_{\Psi_{2}}$ such that the probability that $\mathrm{L}\left(\mathrm{S}\left(\Delta e_{\Psi}\right)\right) \rightarrow \Delta e_{\Psi_{2}}$ is $2^{-6}$, and exist $126 e_{\Psi_{2}}$ such that the probability that $\mathrm{L}\left(\mathrm{S}\left(\Delta e_{\Psi}\right)\right) \rightarrow \Delta e_{\Psi_{2}}$ is $2^{-7}$. Due to the L transformation, the four 32-bit words in any $e_{\Psi}$ are all nonzero. Thus, for any $e_{\Psi_{2}}$, if we define the Event A: $\left(\mathrm{L}\left(\mathrm{S}\left(\Delta e_{\Psi_{2}}\right)\right) \oplus\right.$ $\left.e_{\Psi}\right) \rightarrow \Delta e_{\Psi_{3}}$, then we can learn that there exists one possible $e_{\Psi_{3}}$ with probability $2^{-24}$, and exist $\left(\begin{array}{l}4 \\ 3\end{array}\right) \cdot 126$ possible $e_{\Psi_{3}}$ with probability $2^{-25},\left(\begin{array}{l}4 \\ 2\end{array}\right) \cdot 126^{2}$ possible $e_{\Psi_{3}}$ with probability $2^{-26},\left(\begin{array}{l}4 \\ 1\end{array}\right) \cdot 126^{3}$ possible $e_{\Psi_{3}}$ with probability $2^{-27}$ and $126^{4}$ possible $e_{\Psi_{3}}$ with probability $2^{-28}$. Consequently, for any $e_{\Psi_{2}}$ and $e_{\Psi_{3}}$, if we 
define the Event $\mathrm{B}:\left(\mathrm{L}\left(\mathrm{S}\left(\Delta\left(e_{\Psi} \oplus e_{\Psi_{2}} \oplus e_{\Psi_{3}}\right)\right)\right) \oplus e_{\Psi}\right) \rightarrow \Delta e_{\Psi_{4}}$, then there exists one possible $e_{\Psi_{4}}$ with probability $2^{-24}$, and exist $\left(\begin{array}{l}4 \\ 3\end{array}\right) \cdot 126$ possible $e_{\Psi_{4}}$ with probability $2^{-25},\left(\begin{array}{l}4 \\ 2\end{array}\right) \cdot 126^{2}$ possible $e_{\Psi_{4}}$ with probability $2^{-26},\left(\begin{array}{l}4 \\ 1\end{array}\right) \cdot 126^{3}$ possible $e_{\Psi_{4}}$ with probability $2^{-27}$ and $126^{4}$ possible $e_{\Psi_{4}}$ with probability $2^{-28}$. Therefore, we can compute a square sum of at least $\left(2^{-6}\right)^{2} \cdot\left[\left(2^{-6}\right)^{2}+126 \cdot\left(2^{-7}\right)^{2}\right] \cdot\left[1 \cdot\left(2^{-24}\right)^{2}+\left(\begin{array}{l}4 \\ 3\end{array}\right)\right.$. $\left.126 \cdot\left(2^{-25}\right)^{2}+\left(\begin{array}{l}4 \\ 2\end{array}\right) \cdot 126^{2} \cdot\left(2^{-26}\right)^{2}+\left(\begin{array}{l}4 \\ 1\end{array}\right) \cdot 126^{3} \cdot\left(2^{-27}\right)^{2}+126^{4} \cdot\left(2^{-28}\right)^{2}\right]^{3} \approx 2^{-109.64}$.

For the 4-round differentials $\gamma \rightarrow \delta^{\prime}$, as mentioned earlier, there are 127 possible $e_{\Phi_{2}}, 1$ possibility with probability $2^{-6}$ and each of the other 126 possibilities with probability $2^{-7}$, thus, this 12 -round rectangle distinguisher has a probability of at least $2^{-109.64} \cdot\left[\left(1 \cdot 2^{-6}+126 \cdot 2^{-7}\right)\right]^{2} \cdot 2^{-128} \approx 2^{-237.64}$ for the correct key, while it has a probability of $\left(2^{-128} \cdot 127\right)^{2} \approx 2^{-242.02}$ for a wrong key.

The 12-round distinguisher can be used to mount a rectangle attack on 14round SMS4. Without loss of generality, we assume the attacked 14 rounds are the first 14 rounds from Rounds 0 to 13. Given the 127 input differences $\left(e_{\Phi}, e_{\Phi}, e_{\Phi}, e_{\Phi_{2}}\right)$ to Round 12 , there are at most $127^{5}$ possible output differences $\left\{\left(e_{\Phi}, e_{\Phi}, e_{\Phi_{2}}, e_{\Phi_{3}}\right) \mid e_{\Phi_{3}} \in \Upsilon\left(e_{\Phi}, e_{\Phi_{2}}\right)\right\}$ just after Round 12, and at most $127^{9}$ possible output differences $\left\{\left(e_{\Phi}, e_{\Phi_{2}}, e_{\Phi_{3}}, e_{\Phi_{4}}\right) \mid e_{\Phi_{3}} \in \Upsilon\left(e_{\Phi}, e_{\Phi_{2}}\right), e_{\Phi_{4}} \in\right.$ $\left.\Pi\left(e_{\Phi}, e_{\Phi_{2}}, e_{\Phi_{3}}\right)\right\}$ just after Round 13 .

As mentioned in the Introduction, our rectangle attack, as well as the impossible differential attack in the next section, uses the early abort technique introduced in 111213; the main idea of the early abort technique is to partially determine whether or not a candidate quartet in a rectangle attack (or a candidate pair in an impossible differential attack) is valid earlier than usual, by guessing only a small fraction of subkeys required; if not, we can discard it immediately, which results in less computations in the left steps and may allow us to break more rounds by guessing the subkeys involved, depending on how many candidates are remaining.

The attack procedure is as follows.

\subsection{Attack Procedure}

1. Choose $2^{120.82}$ pairs of plaintexts $\left(P_{i}, \widetilde{P}_{i}\right)$ with difference $\left(e_{\Psi_{1}}, e_{\Psi}, e_{\Psi}, e_{\Psi}\right)$, $i=1,2, \cdots, 2^{120.82}$. In a chosen-plaintext attack scenario, obtain their corresponding ciphertext pairs; we denote them by $\left(C_{i}, \widetilde{C}_{i}\right)$, respectively. These ciphertext pairs generate about $2^{120.82 \times 2} / 2=2^{240.64}$ candidate quartets $\left(\left(C_{i_{1}}, \widetilde{C}_{i_{1}}\right),\left(C_{i_{2}}, \widetilde{C}_{i_{2}}\right)\right)$, for $1 \leq i_{1} \leq i_{2} \leq 2^{120.82}$. We only choose those such that both $C_{i_{1}} \oplus C_{i_{2}}$ and $\widetilde{C}_{i_{1}} \oplus \widetilde{C}_{i_{2}}$ belong to $\left\{\left(e_{\Phi}, e_{\Phi_{2}}, e_{\Phi_{3}}, e_{\Phi_{4}}\right) \mid e_{\Phi_{3}} \in\right.$ $\left.\Upsilon\left(e_{\Phi}, e_{\Phi_{2}}\right), e_{\Phi_{4}} \in \Pi\left(e_{\Phi}, e_{\Phi_{2}}, e_{\Phi_{3}}\right)\right\}$.

2. For all the remaining quartets $\left(\left(C_{i_{1}}, \widetilde{C}_{i_{1}}\right),\left(C_{i_{2}}, \widetilde{C}_{i_{2}}\right)\right)$, do as follows.

(a) For $\left(C_{i_{1}}, C_{i_{2}}\right)$, compute the four-byte difference of their intermediate values just before the $\mathrm{L}$ transformation in Round 13; we denote them by $\left(\Delta_{i_{1}, i_{2}, 0}^{13}, \Delta_{i_{1}, i_{2}, 1}^{13}, \Delta_{i_{1}, i_{2}, 2}^{13}, \Delta_{i_{1}, i_{2}, 3}^{13}\right)$, respectively. For $\left(\widetilde{C}_{i_{1}}, \widetilde{C}_{i_{2}}\right)$, compute the four-byte difference of their intermediate values just before the $\mathrm{L}$ transformation in Round 13 ; we denote them by $\left(\widetilde{\Delta}_{i_{1}, i_{2}, 0}^{13}, \widetilde{\Delta}_{i_{1}, i_{2}, 1}^{13}, \widetilde{\Delta}_{i_{1}, i_{2}, 2}^{13}\right.$, $\left.\widetilde{\Delta}_{i_{1}, i_{2}, 3}^{13}\right)$, respectively. 
(b) For $j=0$ to 3: Guess the $j$-th byte $R K_{13, j}$ of the subkey $R K_{13}$ in Round 13 , and partially decrypt every remaining quartet $\left(\left(C_{i_{1}}, C_{i_{2}}\right),\left(\widetilde{C}_{i_{1}}, \widetilde{C}_{i_{2}}\right)\right)$ with $R K_{13, j}$ to get the $j$-th bytes of their intermediate values just after the $\mathrm{S}$ transformation in Round 13 ; we denote them by $\left(\left(T_{i_{1}, j}, T_{i_{2}, j}\right)\right.$, $\left.\left(\widetilde{T}_{i_{1}, j}, \widetilde{T}_{i_{2}, j}\right)\right)$, respectively. Finally, check if $T_{i_{1}, j} \oplus T_{i_{2}, j}=\Delta_{i_{1}, i_{2}, j}^{13}$ and $\widetilde{T}_{i_{1}, j} \oplus \widetilde{T}_{i_{2}, j}=\widetilde{\Delta}_{i_{1}, i_{2}, j}^{13}$. If 6 or more quartets pass this test, execute next with them, (otherwise, repeat this iteration with another key guess).

Finally, for every remaining $\left(\left(C_{i_{1}}, \widetilde{C}_{i_{1}}\right),\left(C_{i_{2}}, \widetilde{C}_{i_{2}}\right)\right)$ we get their intermediate values just after Round 12 ; we denote them by $\left(\left(T_{i_{1}}, \widetilde{T}_{i_{1}}\right),\left(T_{i_{2}}, \widetilde{T}_{i_{2}}\right)\right)$, respectively.

3. For all the quartets $\left(\left(T_{i_{1}}, \widetilde{T}_{i_{1}}\right),\left(T_{i_{2}}, \widetilde{T}_{i_{2}}\right)\right)$, do as follows.

(a) For $\left(T_{i_{1}}, T_{i_{2}}\right)$, compute the four-byte difference of their intermediate values just before the $\mathrm{L}$ transformation in Round 12 ; we denote them by $\left(\Delta_{i_{1}, i_{2}, 0}^{12}, \Delta_{i_{1}, i_{2}, 1}^{12}, \Delta_{i_{1}, i_{2}, 2}^{12}, \Delta_{i_{1}, i_{2}, 3}^{12}\right)$, respectively. For $\left(\widetilde{T}_{i_{1}}, \widetilde{T}_{i_{2}}\right)$, compute the four-byte difference of their intermediate values just before the $\mathrm{L}$ transformation in Round 12 ; we denote them by $\left(\widetilde{\Delta}_{i_{1}, i_{2}, 0}^{12}, \widetilde{\Delta}_{i_{1}, i_{2}, 1}^{12}, \widetilde{\Delta}_{i_{1}, i_{2}, 2}^{12}\right.$, $\left.\widetilde{\Delta}_{i_{1}, i_{2}, 3}^{12}\right)$, respectively.

(b) For $j=0$ to 3: Guess the $j$-th byte $R K_{12, j}$ of the subkey $R K_{12}$ in Round 12 , partially decrypt every quartet $\left(\left(T_{i_{1}}, T_{i_{2}}\right),\left(\widetilde{T}_{i_{1}}, \widetilde{T}_{i_{2}}\right)\right)$ with $R K_{12, j}$ to get the $j$-th bytes of their intermediate values just after the $S$ transformation in Round 12; we denote them by $\left(\left(Q_{i_{1}, j}, Q_{i_{2}, j}\right),\left(\widetilde{Q}_{i_{1}, j}, \widetilde{Q}_{i_{2}, j}\right)\right)$, respectively. Finally, check if $Q_{i_{1}, j} \oplus Q_{i_{2}, j}=\Delta_{i_{1}, i_{2}, j}^{12}$ and $\widetilde{Q}_{i_{1}, j} \oplus \widetilde{Q}_{i_{2}, j}=$ $\widetilde{\Delta}_{i_{1}, i_{2}, j}^{12}$. If 6 or more quartets pass this test, execute next with them, (otherwise, repeat this iteration with another key guess).

4. For every $\left(R K_{12}, R K_{13}\right)$ passing Step 3, we can deduce that there are at most $2^{64}$ possible 128 -bit user keys from these two 32 -bit subkeys. Then, we do a trial encryption with one known pair of plaintext and ciphertext. If a 128-bit key is suggested, output it as the user key of the 14-round SMS4; otherwise, go to Step 2-(b).

To produce a difference $\left(e_{\Phi}, e_{\Phi}, e_{\Phi}, e_{\Phi_{2}}\right)$ just before Round 12 , the two ciphertext pairs in a right quartet must have differences belonging to the set $\left\{\left(e_{\Phi}, e_{\Phi_{2}}, e_{\Phi_{3}}, e_{\Phi_{4}}\right) \mid e_{\Phi_{3}} \in \Upsilon\left(e_{\Phi}, e_{\Phi_{2}}\right), e_{\Phi_{4}} \in \Pi\left(e_{\Phi}, e_{\Phi_{2}}, e_{\Phi_{3}}\right)\right\}$, so a candidate quartet that does not meet this filtering condition is an incorrect quartet. As a result, only about $2^{240.64} \cdot\left(\frac{127^{9}}{2^{128}}\right)^{2} \approx 2^{110.46}$ candidate quartets are chosen in Step 1 .

In Steps 2-(b) and 3-(b), a candidate quartet passes every test with a probability of $\left(\frac{1}{127}\right)^{2} \approx 2^{-13.98}$, and the number of the pairs passing every step has a binomial distribution, so it is expected that almost all the $2^{56}$ guesses of $\left(R K_{12,0}, R K_{12,1}, R K_{12,2}, R K_{13,0}, R K_{13,1}, R K_{13,2}, R K_{13,3}\right)$ will pass the test with $j=2$ in Step 3 -(b), and for every guess about $2^{110.46} \cdot 2^{-13.98 \times 7}=2^{12.6}$ candidate quartets are expected to remain after the test with $j=2$ in Step 3(b). In the test with $j=3$ in Step 3-(b), the probability that 6 or more quartets pass the tests for a wrong guess is approximately $\sum_{i=6}^{2^{12.6}}\left[\left(\begin{array}{c}2^{12.6} \\ i\end{array}\right) \cdot\left(2^{-13.98}\right)^{i} \cdot(1-\right.$ $\left.\left.2^{-13.98}\right)^{2^{12.6}-i}\right] \approx 2^{-17.77}$, thus it is expected that about $2^{64} \cdot 2^{-17.77}=2^{46.23}$ 
Table 3. The two 6-round differentials in the 12-round impossible differentials, where $x_{i} \in \Theta\left(e_{\Gamma}\right), y_{i} \in \Upsilon\left(e_{\Gamma}, x_{i}\right), z_{i} \in \Pi\left(e_{\Gamma}, x_{i}, y_{i}\right),(i=1,2)$

\begin{tabular}{|c|cccc||cc|ccc|}
\hline Round $(i) \downarrow$ & $\Delta X_{i, 0}$ & $\Delta X_{i, 1}$ & $\Delta X_{i, 2}$ & $\Delta X_{i, 3}$ & $\operatorname{Round}(i) \uparrow$ & $\Delta X_{i, 0}$ & $\Delta X_{i, 1}$ & $\Delta X_{i, 2}$ & $\Delta X_{i, 3}$ \\
\hline \hline 0 & $e_{\Gamma}$ & $e_{\Gamma}$ & $e_{\Gamma}$ & 0 & 6 & $z_{2}$ & $y_{2}$ & $x_{2}$ & $e_{\Gamma}$ \\
\hline 1 & $e_{\Gamma}$ & $e_{\Gamma}$ & 0 & $e_{\Gamma}$ & 7 & $y_{2}$ & $x_{2}$ & $e_{\Gamma}$ & $e_{\Gamma}$ \\
\hline 2 & $e_{\Gamma}$ & 0 & $e_{\Gamma}$ & $e_{\Gamma}$ & 8 & $x_{2}$ & $e_{\Gamma}$ & $e_{\Gamma}$ & $e_{\Gamma}$ \\
\hline 3 & 0 & $e_{\Gamma}$ & $e_{\Gamma}$ & $e_{\Gamma}$ & 9 & $e_{\Gamma}$ & $e_{\Gamma}$ & $e_{\Gamma}$ & 0 \\
\hline 4 & $e_{\Gamma}$ & $e_{\Gamma}$ & $e_{\Gamma}$ & $x_{1}$ & 10 & $e_{\Gamma}$ & $e_{\Gamma}$ & 0 & $e_{\Gamma}$ \\
\hline 5 & $e_{\Gamma}$ & $e_{\Gamma}$ & $x_{1}$ & $y_{1}$ & 11 & $e_{\Gamma}$ & 0 & $e_{\Gamma}$ & $e_{\Gamma}$ \\
\hline output & $e_{\Gamma}$ & $x_{1}$ & $y_{1}$ & $z_{1}$ & output & 0 & $e_{\Gamma}$ & $e_{\Gamma}$ & $e_{\Gamma}$ \\
\hline
\end{tabular}

guesses of $\left(R K_{12}, R K_{13}\right)$ are suggested after the test with $j=3$ in Step $3-(\mathrm{b})$. In Step 4 , the expected number of wrong 128 -bit keys is about $2^{-128} \cdot 2^{46.23+64}=$ $2^{-17.77}$, which is very low.

The attack requires $2^{121.82}$ chosen plaintexts. The required memory space is dominated by the ciphertexts, which is about $2^{121.82} \cdot 16=2^{125.82}$ memory bytes. The time complexity of Steps $2-4$ is dominated by the partial decryptions for $j=0$ in Step 2 -(b), which is about $4 \cdot 2^{8} \cdot 2^{110.46} \cdot \frac{1}{14} \approx 2^{116.66} 14$-round SMS4 computations.

As the probability of the distinguisher is $2^{-237.64}$, it is expect there are $8\left(=2^{240.64} \cdot 2^{-237.64}\right)$ right quartets for the correct key in Step 3-(c). The probability that 6 or more quartets pass the test in Step 3-(c) for the correct subkeys is approximately $\sum_{i=6}^{2^{240.64}}\left[\left(\begin{array}{c}2^{240.64} \\ i\end{array}\right) \cdot\left(2^{-237.64}\right)^{i} \cdot\left(1-2^{-237.64}\right)^{2^{240.64}-i}\right] \approx 0.8$, therefore, with a success probability of $80 \%$, this related-key rectangle attack can break 14-round SMS4, faster than an exhaustive key search.

\section{Impossible Differential Attack on 16-Round SMS4}

An impossible differential [2] is a differential [5] with a zero probability; that is, it would never happen under any situation.

In this section, we exploit certain 12-round impossible differentials in SMS4. Finally, we show that impossible differential cryptanalysis can break SMS4 reduced to 16 rounds.

\subsection{2-Round Impossible Differentials}

The 12-round impossible differentials are $\left(e_{\Gamma}, e_{\Gamma}, e_{\Gamma}, 0\right) \nrightarrow\left(0, e_{\Gamma}, e_{\Gamma}, e_{\Gamma}\right)$, where $\Gamma$ is defined as an arbitrary but nonempty subset of the set $\{0,1, \cdots, 15\}$. These 12-round impossible differentials are built in a miss-in-the-middle manner [3]: a 6-round differential with probability 1 is concatenated with another 6 -round differential with probability 1 , but the intermediate differences of these two differentials contradict one another. See Table 3 .

The first 6-round differential with probability 1 is $\left(e_{\Gamma}, e_{\Gamma}, e_{\Gamma}, 0\right) \rightarrow\left(e_{\Gamma}, ?, ?\right.$, $?)$. The input difference $\left(e_{\Gamma}, e_{\Gamma}, e_{\Gamma}, 0\right)$ to Round 0 propagates with probability 1 
to the difference $\left(e_{\Gamma}, e_{\Gamma}, 0, e_{\Gamma}\right)$ after one round, which then propagates with a 1 probability to the difference $\left(0, e_{\Gamma}, e_{\Gamma}, e_{\Gamma}\right)$ after the following two rounds. Then, the difference $\left(0, e_{\Gamma}, e_{\Gamma}, e_{\Gamma}\right)$ definitely propagates to a difference belonging to the set $\left\{\left(e_{\Gamma}, e_{\Gamma}, e_{\Gamma}, x_{1}\right) \mid x_{1} \in \Theta\left(e_{\Gamma}\right)\right\}$ after Round 3 , which finally propagates with probability 1 to a difference belonging to $\left\{\left(e_{\Gamma}, x_{1}, y_{1}, z_{1}\right) \mid x_{1} \in \Theta\left(e_{\Gamma}\right), y_{1} \in\right.$ $\left.\Upsilon\left(e_{\Gamma}, x_{1}\right), z_{1} \in \Pi\left(e_{\Gamma}, x_{1}, y_{1}\right)\right\}$ after Rounds 4 and 5. On the other hand, when we roll back the output difference $\left(0, e_{\Gamma}, e_{\Gamma}, e_{\Gamma}\right)$ of the second 6 -round differential through the three consecutive rounds from Rounds 9 to 11 in the reverse direction, we will get the difference $\left(e_{\Gamma}, e_{\Gamma}, e_{\Gamma}, 0\right)$ just before Round 9 with probability 1 . Then, when we roll back the difference $\left(e_{\Gamma}, e_{\Gamma}, e_{\Gamma}, 0\right)$ through Round 8, we will definitely get a difference belonging to the set $\left\{\left(x_{2}, e_{\Gamma}, e_{\Gamma}, e_{\Gamma}\right)\right.$ $\left.x_{2} \in \Theta\left(e_{\Gamma}\right)\right\}$. Finally, when we continue to go back for two more rounds, we can definitely get a difference belonging to the set $\left\{\left(z_{2}, y_{2}, x_{2}, e_{\Gamma}\right) \mid x_{2} \in \Theta\left(e_{\Gamma}\right), y_{2} \in\right.$ $\left.\Upsilon\left(e_{\Gamma}, x_{2}\right), z_{2} \in \Pi\left(e_{\Gamma}, x_{2}, y_{2}\right)\right\}$ just before Round 6. Now, a contradiction occurs, for we never get the one-round output difference $\left\{\left(y_{2}, x_{2}, e_{\Gamma}, e_{\Gamma}\right) \mid x_{2} \in\right.$ $\left.\Theta\left(e_{\Gamma}\right), y_{2} \in \Upsilon\left(e_{\Gamma}, x_{2}\right)\right\}$ given an input difference belonging to $\left\{\left(e_{\Gamma}, x_{1}, y_{1}, z_{1}\right) \mid\right.$ $\left.x_{1} \in \Theta\left(e_{\Gamma}\right), y_{1} \in \Upsilon\left(e_{\Gamma}, x_{1}\right), z_{1} \in \Pi\left(e_{\Gamma}, x_{1}, y_{1}\right)\right\}$. More specifically, to get a one-round output difference belonging to $\left\{\left(y_{2}, x_{2}, e_{\Gamma}, e_{\Gamma}\right) \mid x_{2} \in \Theta\left(e_{\Gamma}\right), y_{2} \in\right.$ $\left.\Upsilon\left(e_{\Gamma}, x_{2}\right)\right\}$, the input difference of the second 6 -round differential should belong to the set $\left\{\left(z_{2}, y_{2}, x_{2}, e_{\Gamma}\right) \mid x_{2} \in \Theta\left(e_{\Gamma}\right), y_{2} \in \Upsilon\left(e_{\Gamma}, x_{2}\right), z_{2} \in \Pi\left(e_{\Gamma}, x_{2}, y_{2}\right)\right\}$, however, note that the output difference of the first 6 -round differential is $\left\{\left(e_{\Gamma}, x_{1}\right.\right.$, $\left.\left.y_{1}, z_{1}\right) \mid x_{1} \in \Theta\left(e_{\Gamma}\right), y_{1} \in \Upsilon\left(e_{\Gamma}, x_{1}\right), z_{1} \in \Pi\left(e_{\Gamma}, x_{1}, y_{1}\right)\right\}$, so it is a necessary that the following five conditions should hold for some sextuple $\left(x_{1}, y_{1}, z_{1}, x_{2}, y_{2}, z_{2}\right)$, where $x_{1}, x_{2} \in \Theta\left(e_{\Gamma}\right), y_{1} \in \Upsilon\left(e_{\Gamma}, x_{1}\right), y_{2} \in \Upsilon\left(e_{\Gamma}, x_{2}\right), z_{1} \in \Pi\left(e_{\Gamma}, x_{1}, y_{1}\right)$ and $z_{2} \in \Pi\left(e_{\Gamma}, x_{2}, y_{2}\right)$ :

$$
\begin{aligned}
& x_{2}=y_{1}, \\
& y_{2}=x_{1}, \\
& z_{1}=e_{\Gamma}, \\
& z_{2}=e_{\Gamma}, \\
& \mathrm{L}\left(\mathrm{S}\left(x_{1} \oplus y_{1} \oplus e_{\Gamma}\right)\right) \oplus e_{\Gamma}=e_{\Gamma} .
\end{aligned}
$$

By Properties 1 and 2 in Section 3, we can learn that Eq. (5) is equivalent to the following equation:

$$
x_{1} \oplus y_{1} \oplus e_{\Gamma}=0 .
$$

We perform a computer search over all the possibilities that may satisfy Eqs. (1)-(4) and (6), but find that there does not exist such a qualified sextuple $\left(x_{1}, y_{1}, z_{1}, x_{2}, y_{2}, z_{2}\right)$ for any nonempty subset $\Gamma$ of the set $\{0,1, \cdots, 15\}$. Thus, these 12-round impossible differentials are impossible.

Before further proceeding, we would like to give the following two remarks: i) We did not check whether there also exist similar 12-round impossible differentials if $\Gamma$ is defined as an arbitrary but nonempty subset of the set $\{0,1, \cdots, 31\}$ (excluding those described above), for this is much more time-consuming due to a sharp increase on the number of the possible differences. It is reasonably 
thought that there also exist similar 12-round impossible differentials for them. ii) We did not check whether one or more of the 12-round impossible differentials can be extended to 13-round impossible differentials by appending one-round differential $\left(e_{\Gamma}, x_{1}, y_{1}, z_{1}\right) \rightarrow\left(x_{1}, y_{1}, z_{1}, ?\right)$ after the first 6 -round differential or one-round differential $\left(?, z_{2}, y_{2}, x_{2}\right) \rightarrow\left(z_{2}, y_{2}, x_{2}, e_{\Gamma}\right)$ before the second 6 -round differential; as there are so many possibilities (some may be identical) for any $\Gamma$ that we do not have an enough powerful computer/workstation on our hands to check these possibilities with a bearable running time.

We can use a 12-round impossible differential to conduct an impossible differential attack on SMS4 reduced to 16 rounds, by taking advantage of the early abort technique introduced in 13 . We assume the attacked 16 rounds are from Rounds 0 to 15 . To reduce the data and time complexities of the attack, we choose $\Gamma=\{0,1, \cdots, 15\}$. We use the 12-round impossible differential from Rounds 2 to 13 . Given the output difference $\left(e_{0,1, \cdots, 15}, e_{0,1, \cdots, 15}, e_{0,1, \cdots, 15}, 0\right)$ of Round 1 , there are $127^{2}$ possible input differences to Round 1 , and at most $127^{6}$ possible input differences to Round 0 ; we denote them by the set $\Sigma_{1}$. Given the input difference $\left(0, e_{0,1, \cdots, 15}, e_{0,1, \cdots, 15}, e_{0,1, \cdots, 15}\right)$ to Round 14 , there are at most $127^{2}$ possible output differences just after Round 14 , and at most $127^{6}$ possible output differences just after Round 15; we denote them by the set $\Sigma_{2}$. The attack procedure is as follows.

\subsection{Attack Procedure}

1. Select $2^{9}$ structures of $2^{96}$ plaintexts each, where the most significant 16 bits of the rightmost two words of the plaintexts in a structure are fixed to certain values, and all the other 96 bit positions take all the possible values. Each structure generates $\left(2^{96} / 2\right)^{2}=2^{190}$ plaintext pairs $\left(P_{i}, P_{j}\right)$ with difference $\left(?, ?, e_{0,1}, \cdots, 15, e_{0,1, \cdots, 15}\right)$; thus, the $2^{9}$ structures propose $2^{199}$ plaintext pairs with difference $\left(?, ?, e_{0,1}, \cdots, 15, e_{0,1, \cdots, 15}\right)$. In a chosen-plaintext attack scenario, obtain all the ciphertexts of $P_{i}$ and $P_{j}$; we denote them by $C_{i}$ and $C_{j}$, respectively. Choose only the ciphertext pairs $\left(C_{i}, C_{j}\right)$ such that $P_{i} \oplus P_{j} \in \Sigma_{1}$ and $C_{i} \oplus C_{j} \in \Sigma_{2}$.

2. For all the remaining pairs $\left(C_{i}, C_{j}\right)$, compute the four-byte difference of their intermediate values just before the $\mathrm{L}$ transformation in Round 15; we denote them by $\left(\Delta_{i, j, 0}^{15}, \Delta_{i, j, 1}^{15}, \Delta_{i, j, 2}^{15}, \Delta_{i, j, 3}^{15}\right)$, respectively. Do as follows.

(a) For $l=0$ to 3: Guess the $l$-th byte $R K_{15, l}$ of the subkey $R K_{15}$ in Round 15, partially decrypt $\left(C_{i}, C_{j}\right)$ with $R K_{15, l}$ to get the $l$-th bytes of their intermediate values just after the $\mathrm{S}$ transformation in Round 15; we denote them by $\left(T_{i, l}, T_{j, l}\right)$, respectively, and keep the pairs such that $T_{i, l} \oplus T_{j, l}=\Delta_{i, j, l}^{15}$.

Finally, for every remaining $\left(C_{i}, C_{j}\right)$ we can get their intermediate values just after Round 14 under the guess for $R K_{15}$; we denote them by $\left(T_{i}, T_{j}\right)$, respectively. 
(b) For all the remaining pairs $\left(T_{i}, T_{j}\right)$, compute the four-byte difference of their intermediate values just before the $\mathrm{L}$ transformation in Round 14; we denote the first two bytes by $\left(\Delta_{i, j, 0}^{14}, \Delta_{i, j, 1}^{14}\right)$, respectively.

(c) For $l=0$ to 1: Guess the $l$-th byte $R K_{14, l}$ of the subkey $R K_{14}$ in Round 14, partially decrypt $\left(T_{i}, T_{j}\right)$ with $R K_{14, l}$ to get the $l$-th bytes of their intermediate values just after the $\mathrm{S}$ transformation in Round 14; we denote them by $\left(Q_{i, l}, Q_{j, l}\right)$, respectively, and keep only the pairs such that $Q_{i, l} \oplus Q_{j, l}=\Delta_{i, j, l}^{14}$.

3. For all the plaintext pairs $\left(P_{i}, P_{j}\right)$ corresponding to the remaining ciphertext pairs $\left(C_{i}, C_{j}\right)$ after Step 2-(c), compute the four-byte difference of their intermediate values just before the $\mathrm{L}$ transformation in Round 0; we denote them by $\left(\Delta_{i, j, 0}^{0}, \Delta_{i, j, 1}^{0}, \Delta_{i, j, 2}^{0}, \Delta_{i, j, 3}^{0}\right)$, respectively. Do as follows.

(a) For $l=0$ to 3: Guess the $l$-th byte $R K_{0, l}$ of the subkey $R K_{0}$ in Round 0 , partially decrypt $\left(P_{i}, P_{j}\right)$ with $R K_{0, l}$ to get the $l$-th bytes of their intermediate values just after the $\mathrm{S}$ transformation in Round 0 ; we denote them by $\left(R_{i, l}, R_{j, l}\right)$, respectively, and keep only the pairs such that $R_{i, l} \oplus$ $R_{j, l}=\Delta_{i, j, l}^{0}$.

Finally, for every remaining $\left(P_{i}, P_{j}\right)$ we can get their intermediate values just after Round 0 under the guess for $R K_{0}$; we denote them by $\left(R_{i}, R_{j}\right)$, respectively.

(b) For all the remaining pairs $\left(R_{i}, R_{j}\right)$, compute the four-byte difference of their intermediate values just before the $\mathrm{L}$ transformation in Round 1; we denote the first two bytes by $\left(\Delta_{i, j, 0}^{1}, \Delta_{i, j, 1}^{1}\right)$, respectively.

(c) Guess the first byte $R K_{1,0}$ of the subkey $R K_{1}$ in Round 1 , and partially decrypt $\left(R_{i}, R_{j}\right)$ with $R K_{1,0}$ to get the first bytes of their intermediate values just after the $\mathrm{S}$ transformation in Round 1; we denote them by $\left(S_{i, 0}, S_{j, 0}\right)$, respectively. Keep only the pairs such that $S_{i, 0} \oplus S_{j, 0}=\Delta_{i, j, 0}^{1}$.

(d) Guess the second byte $R K_{1,1}$ of the subkey $R K_{1}$ in Round 1, partially decrypt $\left(R_{i}, R_{j}\right)$ with $R K_{1,1}$ to get the second bytes of their intermediate values just after the $\mathrm{S}$ transformation in Round 1 ; we denote them by $\left(S_{i, 1}, S_{j, 1}\right)$, respectively, and check if $S_{i, 1} \oplus S_{j, 1}=\Delta_{i, j, 1}^{1}$. If there exists a qualified pair, then discard the guess of the 96 subkey bits, and try another; otherwise, record it, and execute Step 4.

4. For a recorded guess of the 96 subkey bits, we can deduce that there are at most $2^{96}$ possible 128 -bit user keys from these two 32 -bit subkeys. Then, we do a trial encryption with one known pair of plaintext and ciphertext. If a 128-bit key is suggested, output it as the user key of the 16-round SMS4; otherwise, go to Step 2-(a).

To get the difference $\left(0, e_{0,1}, \cdots, 15, e_{0,1, \cdots, 15}, e_{0,1, \cdots, 15}\right)$ just before Round 14 a ciphertext pair must have a difference belonging to $\Sigma_{2}$, and its corresponding plaintext pair must have a difference belonging to $\Sigma_{1}$ to get the difference $\left(e_{0,1, \cdots, 15}, e_{0,1}, \cdots, 15, e_{0,1}, \cdots, 15,0\right)$ just before Round 2 , which poses a filtering condition of $\frac{127^{6}}{2^{64}} \cdot \frac{127^{6}}{2^{128}} \approx 2^{-108.12}$ over all the ciphertext pairs. There is a filtering condition of $\frac{1}{127}$ in every test of Steps 2-(a), 2-(c), 3-(a) and 3-(c). Therefore, it is expected that only $2^{13.99}$ pairs pass Step 3-(c) for every guess of 
( $\left.R K_{0}, R K_{1,0}, R K_{14,0}, R K_{14,1}, R K_{15}\right)$, and all these remaining pairs have the difference $\left(0, e_{0,1}, \cdots, 15, e_{0,1, \cdots, 15}, e_{0,1}, \cdots, 15\right)$ just before Round 14. In Step 3-(d), a remaining pair propagates with a probability of $\frac{1}{127}$ to a pair of intermediate values with difference $\left(e_{0,1}, \cdots, 15, e_{0,1}, \cdots, 15, e_{0,1}, \cdots, 15,0\right)$ just after Round 1 , thus, we expect with a probability of $\frac{1}{127}$ to get a pair $\left(S_{i, 1}, S_{j, 1}\right)$ such that $S_{i, 1} \oplus S_{j, 1}=$ $\Delta_{i, j, 1}^{1}$, which means the pair has a difference $\left(e_{0,1, \cdots, 15}, e_{0,1, \cdots, 15}, e_{0,1, \cdots, 15}, 0\right)$ just after Round 1; however, a subkey guess for which there exists such a pair is impossible. Hence, after analysing all the $2^{13.99}$ remaining ciphertext pairs, only $2^{96} \cdot\left(1-2^{-6.99}\right)^{2^{13.99}} \approx 2^{-88.32}$ possible guesses of the 96 subkey bits pass Step 3-(d). As a result, the expected number of wrong 128-bit keys in Step 4 is about $2^{-128} \cdot 2^{96}=2^{-32}$, which is extremely low, so we can find the correct 128 -bit user key.

The attack requires $2^{105}$ chosen plaintexts. The time complexity of Steps $2-4$ is dominated by the partial encryptions/decryptions in Steps 2-(a), 2-(c), 3-(a), 3 - (c) and 3-(d), which is approximately $\sum_{l=1}^{11}\left(2 \cdot 2^{90.88} \cdot 2^{8 \cdot l} \cdot \frac{1}{127^{l-1}} \cdot \frac{1}{16}\right)+2 \cdot 2^{96}$. $\left[1+\left(1-2^{-6.99}\right)+\cdots+\left(1-2^{-6.99}\right)^{2^{13.99}}\right] \cdot \frac{1}{16} \approx 2^{107} 16$-round SMS4 computations.

\section{Concluding Remarks}

In this paper, we analyse the security of the SMS4 block cipher used in WAPI, a Chinese national standard. We present a rectangle attack on SMS4 reduced to 14 rounds and an impossible differential attack on SMS4 reduced to 16 rounds. These are better than any previously known cryptanalytic results on SMS4 in terms of the numbers of attacked rounds.

Like most cryptanalytic results on block ciphers, our attacks are theoretical in the sense of the assumptions of differential cryptanalysis. We stress that our cryptanalytic attacks do not endanger the full 32 round version of SMS4; the 32 rounds provide a sufficient safety margin against our attacks.

\section{Acknowledgments}

The author is very grateful to his supervisor Prof. Chris Mitchell and an anonymous referee for their editorial comments.

\section{References}

1. Office of State Commercial Cryptography Administration.: P.R. China, The SMS4 Block Cipher (in Chinese), Archive available at: http://www.oscca.gov.cn/UpFile/200621016423197990.pdf

2. Biham, E., Biryukov, A., Shamir, A.: Cryptanalysis of Skipjack reduced to 31 rounds using impossible differentials. In: Stern, J. (ed.) EUROCRYPT 1999. LNCS, vol. 1592, pp. 12-23. Springer, Heidelberg (1999)

3. Biham, E., Biryukov, A., Shamir, A.: Miss in the middle attacks on IDEA and Khufu. In: Knudsen, L.R. (ed.) FSE 1999. LNCS, vol. 1636, pp. 124-138. Springer, Heidelberg (1999) 
4. Biham, E., Dunkelman, O., Keller, N.: The rectangle attack — rectangling the Serpent. In: Pfitzmann, B. (ed.) EUROCRYPT 2001. LNCS, vol. 2045, pp. 340357. Springer, Heidelberg (2001)

5. Biham, E., Shamir, A.: Differential cryptanalysis of the Data Encryption Standard. Springer, Heidelberg (1993)

6. The Institute of Electrical and Electronics Engineers (IEEE), http://grouper.ieee.org/groups/802/11

7. International Standardization of Organization (ISO).: International StandardISO/IEC 8802-11: Wireless LAN Medium Access Control (MAC) and Physical Layer (PHY) specifications, http://www.iso.org/iso/en/CatalogueDetailPage. CatalogueDetail?CSNUMBER=39777

8. Kelsey, J., Kohno, T., Schneier, B.: Amplified boomerang attacks against reducedround MARS and Serpent. In: Schneier, B. (ed.) FSE 2000. LNCS, vol. 1978, pp. 75-93. Springer, Heidelberg (2001)

9. Knudsen, L.R., Wagner, D.: Integral cryptanalysis. In: Daemen, J., Rijmen, V. (eds.) FSE 2002. LNCS, vol. 2365, pp. 112-127. Springer, Heidelberg (2002)

10. Liu, F., Ji, W., Hu, L., Ding, J., Lv, S., Pyshkin, A., Weinmann, R.P.: Analysis of the SMS4 block cipher. In: Pieprzyk, J., Ghodosi, H., Dawson, E. (eds.) ACISP 2007. LNCS, vol. 4586, pp. 158-170. Springer, Heidelberg (2007)

11. Lu, J., Kim, J., Keller, N., Dunkelman, O.: Related-key rectangle attack on 42round SHACAL-2. In: Katsikas, S.K., Lopez, J., Backes, M., Gritzalis, S., Preneel, B. (eds.) ISC 2006. LNCS, vol. 4176, pp. 85-100. Springer, Heidelberg (2006)

12. Lu, J., Kim, J., Keller, N., Dunkelman, O.: Differential and rectangle attacks on reduced-round SHACAL-1. In: Barua, R., Lange, T. (eds.) INDOCRYPT 2006. LNCS, vol. 4329, pp. 17-31. Springer, Heidelberg (2006)

13. Lu, J., Kim, J., Keller, N., Dunkelman, O.: Improving the efficiency of impossible differential cryptanalysis of reduced Camellia and MISTY1, Archive available at: http://jiqiang.googlepages.com

14. National Institute of Standards and Technology.: U.S.A., Advanced Encryption Standard (AES) FIPS-197 (2001)

15. Wagner, D.: The boomerang attack. In: Knudsen, L.R. (ed.) FSE 1999. LNCS, vol. 1636, pp. 156-170. Springer, Heidelberg (1999)

16. Zhang, L., Wu, W.: Differential fault attack on SMS4 (in Chinese). Chinese Journal of Computers 29(9) (2006) 